\title{
Bone Formation with Two Types of Grafting Materials: A Histologic and Histomorphometric Study
}

\author{
Amir Reza Rokn ${ }^{1,2}$, Mohammad Amin Khodadoostan ${ }^{3}$, Rasouli Ghahroudi AAR ${ }^{4}$, \\ Pourya Motahhary ${ }^{5}$, Mohammad Javad Kharrazi Fard ${ }^{6}$, De Bruyn $\mathrm{H}^{7}$, Rose Afzalifar ${ }^{8}$, \\ E Soolari ${ }^{9}$ and Ahmad Soolari ${ }^{10_{*}}$
}

\author{
${ }^{1}$ Associate Professor, Department of Periodontics, School of Dentistry, Tehran University of Medical Sciences, \\ Tehran, Iran \\ ${ }^{2}$ Associate Professor, Dental Research Center, Tehran University of Medical Sciences, Tehran, Iran \\ ${ }^{3}$ Assistant Professor, Department of Periodontics, School of Dentistry, Hamadan University of Medical \\ Sciences, Hamadan, Iran \\ ${ }^{4}$ Assistant Professor, Department of Periodontics, School of Dentistry, Tehran University of Medical Sciences, \\ Tehran, Iran \\ ${ }^{5}$ Assistant Professor, Department of Oral Pathology, School of Dentistry, Tehran University of Medical Sciences, \\ Tehran, Iran \\ ${ }^{6}$ Statistical Consultant, Dental Research Center, Tehran University of Medical Sciences, Tehran, Iran \\ ${ }^{7}$ Professor and Chairman, Department of Periodontology and Oral Implantology, Dental School, Faculty of Medicine \\ and Health Sciences, University Ghent Belgium, Ghent, Belgium \\ ${ }^{8}$ Dentist, Dental Research Center, Tehran University of Medical Sciences, Tehran, Iran \\ ${ }^{9}$ College of Chemical and Life Science, University of Maryland, MD USA \\ ${ }^{10}$ Diplomate of American Board of Periodontology, Private practice, Silver Spring, MD, USA
}

\begin{abstract}
Background: Although autogenous bone grafts are considered the gold standard for bone regeneration, they have certain limitations, including patient morbidity at the harvest site. Synthetic bone substitutes have been developed to overcome some of these limitations. The present study aimed to compare the osteogenic properties of Straumann Bone Ceramic (SBC), which is a biphasic calcium phosphate, with Bio-Oss, an inorganic bovine bone material, in an animal model.

Methods: Thirteen rabbits were included in this study. In each rabbit, four 6.5-mm-diameter identical defects were prepared on the calvarium. One site was filled with Bio-Oss, the second site was treated with small-particle SBC, the third site was treated with large-particle SBC, and the fourth site was left as an untreated control. After 4 and 8 weeks, the animals were sacrificed, and histologic and histomorphometric examinations were performed. The data were analyzed using Friedman and multiple-comparison Mann-Whitney $U$ tests.

Results: There were no statistically significant differences in the amount of bone fill between the four groups. L-SBC showed more inflammation and foreign-body reactions than the other bone substitutes.

Conclusion: No statistically significant differences were found between groups. Further studies on this issue seem necessary.
\end{abstract}

Keywords: Bone transplantation, bone ceramics, inorganic bovine bone mineral, animal study.

\section{INTRODUCTION}

The demand for an ideal nonautogenous bone grafting material is increasing due to its unlimited supply, easy storage, and sterility [1]. Synthetic bone replacements

*Address correspondence to this author at the 11616 Toulone Dr., Potomac, MD 20854; Tel: 301 674-9815; Fax: 240845 1087;

E-mail: asoolari@gmail.com (alloplasts) are osteoconductive - that is, they provide a scaffold for bone deposition-as opposed to osteoinductive materials such as autografts, which may include growth factors necessary for osteogenesis. Commercially available synthetic bone replacements have been made of hydroxyapatite, tricalcium phosphate, calcium sulfate, and combinations of these minerals, and fabrication techniques, crystal configurations, pore dimensions, mechanical properties, and resorption rates vary [2]. 


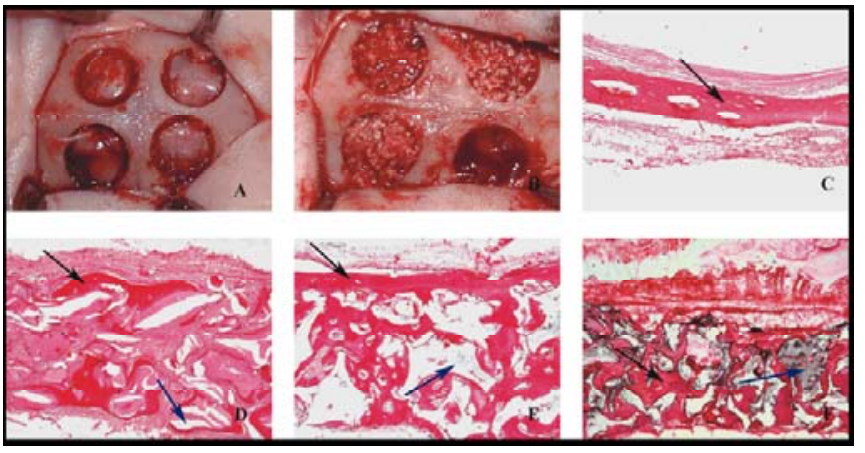

Fig. (1). (a) Four identical defects were prepared in the rabbit calvarium. (b) Three defects were filled with bone xenograft or alloplast materials and one was left unfilled to serve as a control. (c) Bone formation in control sample at $20 \times$ magnification (black arrow $=$ regenerated bone). $(\mathbf{d})$ Bio-Oss sample at $20 \times$ magnification (black arrow $=$ regenerated bone; blue arrow $=$ remaining grafting material). (e) Small-particle bone ceramic sample at $20 \times$ magnification (black arrow $=$ regenerated bone; blue arrow $=$ remaining grafting material). (f) Large-particle bone ceramic sample at $20 \times$ magnification (black arrow $=$ regenerated bone; blue arrow $=$ remaining grafting material) .

Ultimately, the successful repair of bony defects with biomaterials is dependent on sufficient vascularization at the implantation site [3]. Ultrastructural analysis, inorganic chemistry, and new processing modes have been used to develop fully synthetic bone graft materials applicable to the reconstruction of osseous defects [4]. To select the appropriate biomaterial for a given situation, a clinician should consider the physical, chemical, biologic, and processing features of a bone graft. The size and geometry of the bony lesions, as well as the surgical conditions of each patient (e.g., blood supply and mechanical stress), also influence treatment outcomes $[5,6]$. Autogenous grafts are considered the gold standard for bone grafting due to their biologic origin and activity. However, the harvesting of autogenous grafts frequently requires additional surgery, and access to donor sites may be limited; these facts have prompted a search for alternative methods and materials [5].

Allograft materials (processed bone from the same species), such as freeze-dried bone and demineralized freezedried bone, have been used for many decades. However, the possibility of disease transmission, although very low, may lessen the desirability of these materials $[7,8]$.

Alloplastic calcium phosphate bone substitutes, such as hydroxyapatite (HA) and tricalcium phosphate (TCP), have been studied because their composition closely resembles the inorganic phase of bone tissue [9]. HA was used extensively in the 1980s but did not induce proper bone remodeling due to chronic inflammation associated with the very slowly resorbing HA granules, which remained unaltered for an extended period of time. TCP has been shown to have superior resorption characteristics. However, its early resorption, prior to cellular attachment and lymphocytic infiltration, has been associated with unsatisfactory results [4]. The HA/TCP ratio can be manipulated to change the substitution rate and bioactivity of calcium phosphate materials, an advantage that has made them clinically useful in oral and orthopedic surgery [9].

One commonly used bone substitute, Bio-Oss (Geistlich Sons Ltd. Wolhusen, Switzerland), is a xenograft (inorganic bovine bone) that promotes new bone formation, does not interfere with bone healing [10-13], and possesses osteoconductive properties that are superior to those of beta-TCP. Another substitute, Straumann Bone Ceramic (Straumann AG, Basel, Switzerland), is a fully synthetic bone substitute material. It is composed of biphasic calcium phosphate $(60 \%$ HA and $40 \%$ beta-TCP). This combination of a nonabsorbable and bioinert material such as HA with a highly resorbable material (i.e., TCP) [14] is bioactive, bioresorbable, and biocompatible, and it has a high osteoinductive potential $[15,16]$. This material may offer better conditions for bone regeneration when compared to traditional bone substitutes [17]. However, some studies have concluded that this material is only osteoconductive $[18,19]$, similar to Bio-Oss [19].

Despite these developments, an ideal substitute for autologous bone is not yet available. The purpose of this study was to histologically and histomorphometrically evaluate the bone repair quality of Straumann Bone Ceramic (SBC) and Bio-Oss in experimental defects prepared in rabbit calvaria. The main hypothesis of this study was that the higher resorption rate of Straumann Bone Ceramic would promote more bone formation than Bio-Oss.

\section{MATERIALS AND METHODOLOGY}

The experimental alloplasts used in the present study were the large-size bone ceramic (L-SBC), with a particle size of 500 to $1000 \mu \mathrm{m}$, and small-size bone ceramic (SSBC), with a particle size ranging from 400 to $700 \mu \mathrm{m}$. Both materials were made of beta-TCP and HA ( $40 \%$ beta-TCP, $60 \% \mathrm{HA})$. Both the L-SBC and S-SBC materials were sintered at $1100^{\circ} \mathrm{C}$ to $1500^{\circ} \mathrm{C}$ and processed to a final porosity of $90 \%$ and a pore size of 100 to $500 \mu \mathrm{m}$.

After careful planning of an interventional animal study and approval by the Ethical Research Committee of the Tehran University School of Dentistry, 13 white New Zealand rabbits were included in this experimental animal study. The mean weight of the rabbits was $2.5 \mathrm{~kg}$. The rabbits were kept on a uniform standard feeding regimen (BabyRabbitPellets, M-0662, Masterfeeds Division, Maple Leaf Mills Ltd, London, Ontario, Canada) starting 2 weeks prior to the study. The study was performed at the Dental Research Center, School of Dentistry, Tehran University of Medical Sciences.

The rabbits were placed under general anesthesia by injection of $10 \%$ ketamine and $2 \%$ xylazine. The calvarium of each animal was scrubbed with $7 \%$ Betadine (povidoneiodine) and the hair on the surgical area was shaved. The area was then isolated with a sterile drape and scrubbed with 7\% Betadine for 5 minutes. An anteroposterior (craniocaudal) $10-\mathrm{cm}$ straight incision was made. The skin and subcutaneous tissues were elevated with a periosteal elevator. Using an angled handpiece motor and a 6.5-mm-diameter trephine bur, the authors created four identical circular defects in the calvarium (Fig. 1A) under external irrigation with normal saline. Anatomic landmarks were referenced to standardize the location of defects; these included the occipital process and the craniocaudal suture. Three types of materials were used to fill three of the defects: (1) large-size bone ceramic (L-SBC), (2) small-size bone ceramic (S-SBC), and (3) Bio-Oss with a particle size of 250 to $1000 \mu \mathrm{m}$. The fourth defect was left unfilled and served as the control. To eliminate bias in defect location, the defect fill sequence 
(two in frontal bone and two in parietal bone) was varied as follows. In the first rabbit, the defects were treated randomly with the three aforementioned materials and the fourth defect was left unfilled as the control. Then these positions were changed rotationally (clockwise) for the other rabbits (Fig. 1B). All locations were recorded on charts.

Following placement of the biomaterials, the periosteum was sutured into place with 4.0 Vicryl (Ethicon, Somerville, NJ, USA) and the skin of the calvarium was sutured with 3-0 Nylon (Ethicon, Somerville, NJ, USA). The experimental animals were transferred to a room with a constant temperature of $37^{\circ} \mathrm{C}$. To control postoperative pain and swelling, 0.1 $\mathrm{mL}$ of ketoprofen was administered daily for up to 3 consecutive days. In addition, $0.6 \mathrm{~mL}$ of enrofloxacin (Baytril, Bayer Corp, Shawnee, KS, USA) was administered subcutaneously. One group of rabbits was sacrificed 4 weeks after the surgery and the other group was sacrificed after 8 weeks. The animals were sacrificed with an injection of $2 \mathrm{~mL}$ intravenous sodium thiopental into the marginal auricular venules.

Samples were then collected from the surgical areas of the experimental defects. The calvarium was detached from the skull with a saw and fixed in $10 \%$ buffered formalin. The distances from the sagittal suture and the parietooccipital suture had been measured and precisely marked prior to the creation of defects; therefore, the same locations were used in all animals, based on the same distances from those sutures.

Histologic sections were prepared from the defect areas, which included the surrounding natural bone. All samples were prepared for hematoxylin and eosin (H\&E) staining. First, they were fixed in $10 \%$ formalin for 5 days and then they were decalcified with $10 \%$ formic acid (Bayer, Germany) for 5 days. During this process, the acid was changed daily and the degree of decalcification was evaluated manually. After decalcification was complete, the samples were put into lithium carbonate for 5 minutes and then each specimen was divided into two parts at the center and prepared for histologic examination in a routine manner. The samples were put into an automatic autotechnicum machine. In this machine they were put into a series of graded alcohol concentrations for dehydration as well as xylol. After they were mounted in paraffin from the cut side, histologic sections $(5 \mu \mathrm{m})$ were created and stained with $\mathrm{H} \& \mathrm{E}$.

Histologic preparation of all the samples was performed in one laboratory with the same standard technique. Materials and stains were the same for all specimens and were checked regularly based on a national standard program. Although all the samples were not prepared in a single day, they were divided into three groups, each of which was prepared simultaneously. At least five sections were taken from each sample, and all sections were checked precisely. All specimens were observed with a double-head light microscope (BX-41, Olympus, Japan) at $20 \times$ magnification by two blinded oral pathologists, who were not informed about the source of each sample (e.g., S-SBC, control) and who had been previously calibrated in evaluating bone histomorphometric parameters. In the event of disagreement about a sample, the pathologists examined the sample together to reach an agreement about the scores.
Four qualitative variables were examined.

1. The intensity of inflammation, according to lymphocytic infiltration around the bone graft materials in the examined defects. Grade 0 (no inflammation): 0-100 lymphocytes; grade 1 (mild inflammation): 100-500 lymphocytes; grade 2 (moderate inflammation): 500-1000 lymphocytes; and grade 3 (severe inflammation): more than 1000 lymphocytes.

2. Presence of a foreign-body reaction, as manifested by the presence of foreign-body giant cells in a granulomatous response. Grade 0 (no foreign-body reaction): 0 foci; grade 1 (mild foreign-body reaction): 0-10 foci; grade 2 (moderate foreign-body reaction): 10-30 foci; and grade 3 (severe foreign body reaction): more than 20 foci.

3. The amount of regenerated bone. Digital photographs were taken of each sample through a light microscope at $20 \times$ magnification (DP-12, Olympus, Japan). In each case, the photographic field was selected from the middle portion of the prepared calvarial opening. These digital photographs were stored and subsequently analyzed by SigmaScan Pro Image Analysis software, Version 5.0, and Adobe Photoshop CS2. In this analysis, the number of pixels in different parts of the image was calculated digitally, and then the average numbers of pixels of regenerated bone were calculated. The entire surgical site was checked for qualitative measures (the surgical area was easy to locate under the microscope, as it was filled with graft material, and graft material was easily detectable). However, only the central part of each surgical site in each section (consisting of $1 \mathrm{~mm}^{2}$ ) was selected for evaluation of quantitative measures (bone formation and remaining graft material) to minimize the influence of the native bone present around the border of the cavity on any bone repair mechanisms effected through the graft material. The histologic characteristics of bone, connective tissue, and graft materials were completely different, so they were easily differentiated and determined based on their specific characteristics; stain intensity (color threshold) was just one of these parameters. The color thresholds for bone, graft material, and connective tissue were different and the borders of these areas were easily detectable. Based on these factors, the amounts of bone, graft material, and connective tissue were determined separately in the images, calculated digitally by a software program, and expressed in pixels, as determined by the agreement of two observers and measured with the microscope ruler. The staining intensity was evaluated qualitatively and determined by the agreement of two observers.

4. The remaining substitute material was expressed as a number by using the same method described for determining the amount of regenerated bone.

Obtained data were analyzed using SPSS version 16 (SPSS Inc, Chicago, IL, USA). To compare the effects of the type of biomaterials on each of the indices, Friedman nonparametric and post hoc tests (using the Bonferroni correction) were used. Mann-Whitney tests were used to compare 
Table 1. Intensity of Inflammation and Foreign-Body Responses in Each Study Group at 4 and 8 Weeks after Surgery

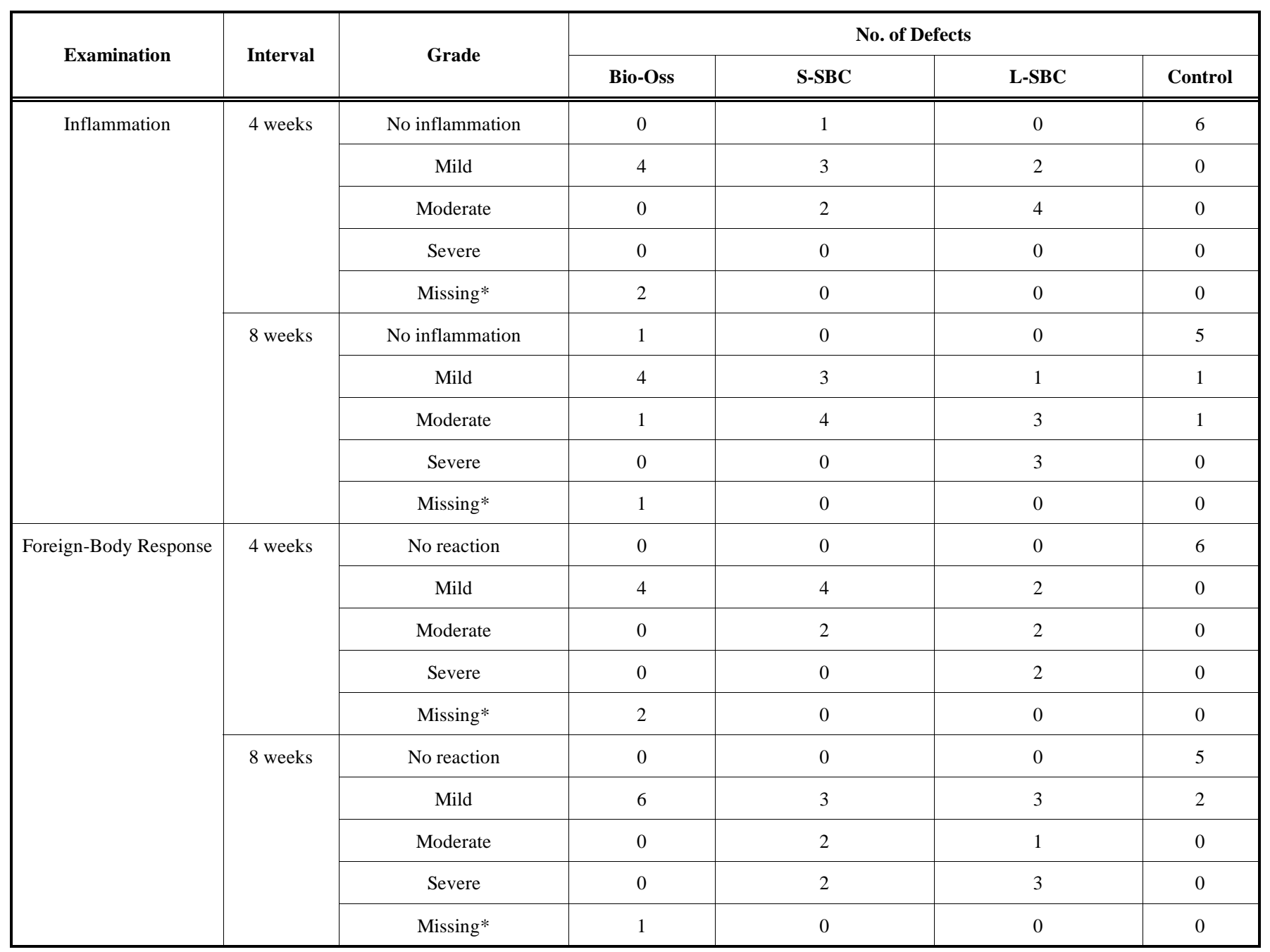

*Sample lost during histologic processing.

Table 2. Mean (SDs) no. of Pixels of Regenerated Bone and Remaining Material in Each Study Sample

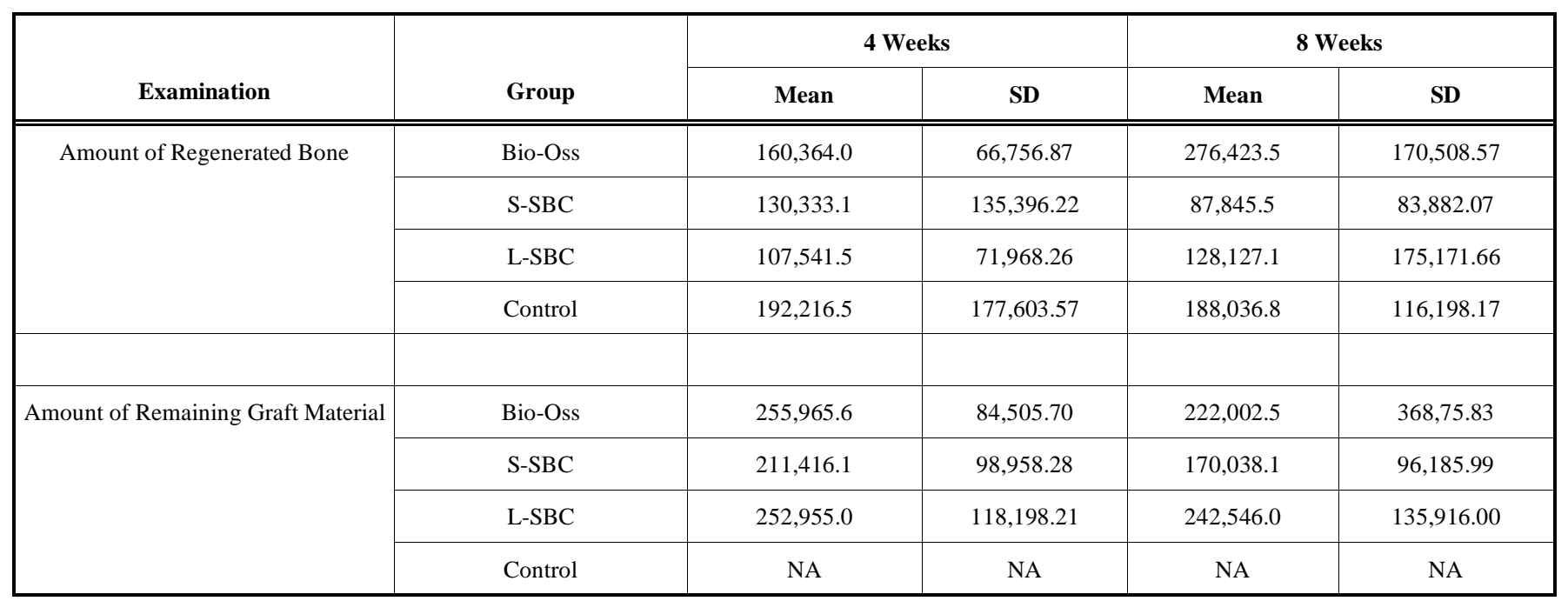

$\mathrm{NA}=$ not applicable. No significant differences were found between materials $(P>.05)$. For Bio-Oss, the amount of regenerated bone was significantly different between week 4 and week $8(P<.05)$. 
Table 3. No. of Defects with Different Amounts of Regenerated Bone and Remaining Biomaterial at Both Time Intervals

\begin{tabular}{|c|c|c|c|c|c|c|}
\hline Examination & Interval & Grade & \multicolumn{4}{|c|}{ Number of Defects } \\
\hline \multirow{6}{*}{ Regenerated Bone } & \multirow{3}{*}{4 weeks } & Few & 1 & 1 & 0 & 3 \\
\hline & & Moderate & 1 & 0 & 3 & 2 \\
\hline & & Missing ${ }^{*}$ & 1 & 0 & 0 & 0 \\
\hline & \multirow[t]{3}{*}{8 weeks } & No or Negligible & 0 & 3 & 3 & 0 \\
\hline & & Few & 0 & 2 & 2 & 3 \\
\hline & & Moderate & 4 & 2 & 2 & 3 \\
\hline \multirow[t]{7}{*}{ Remaining Biomaterial } & \multirow[t]{5}{*}{4 weeks } & No or Negligible & 0 & 0 & 0 & NA \\
\hline & & Few & 0 & 1 & 1 & NA \\
\hline & & Moderate & 0 & 3 & 0 & NA \\
\hline & & High & 4 & 2 & 5 & NA \\
\hline & & Missing ${ }^{*}$ & 2 & 0 & 0 & \\
\hline & \multirow[t]{2}{*}{8 weeks } & No or Negligible & 0 & 0 & 0 & NA \\
\hline & & Few & 0 & 0 & 0 & NA \\
\hline
\end{tabular}

NA = not applicable.

"Sample lost during histologic processing.

No differences were found between groups at any time period $(P>.05)$.

histologic and histomorphometric indices between the two follow-up periods.

\section{RESULTS}

Fifty-two defects, each $6.5 \mathrm{~mm}$ in diameter, were prepared using a trephine bur in the calvaria of 13 rabbits. Each of the three graft materials was used to fill in 13 defects, and 13 defects were left unfilled (controls). Histologic sections were prepared at weeks 4 and 8 and examined microscopically (Figs. 1C to 1F). Six and seven rabbits were sacrificed at weeks 4 and 8 , respectively. Some samples could not be processed histologically and therefore could not be evaluated (Tables 1 to 3 ).

The intensity of inflammation and the foreign-body responses of all specimens were evaluated qualitatively. Table 1 presents the inflammation and foreign-body reactions observed in each study group according to healing time and intensity. The least amount of inflammation was observed in the control group, followed in order by the Bio-Oss, S-SBC, and L-SBC groups. In terms of inflammation, significant differences $(P<.05)$ were found between the control and
Bio-Oss sites, the control and S-SBC sites, the control and LSBC sites, and between the Bio-Oss and L-SBC sites $(P<$ $.05)$. However, no significant differences in inflammation were seen between the L-SBC and S-SBC groups. With regard to the presence of multinucleated giant cells, there were significantly more cells observed for the grafted groups than for the ungrafted control group $(P<.05)$.

The means and standard deviations of the amount of regenerated bone and the amount of substitute material remaining in the defects at each time point are presented quantitatively in Table 2 and qualitatively in Table 3 . The amount of bone formed in the Bio-Oss group was greater than that seen for other groups, although this difference was not statistically significant $(P>.05)$. The control sites showed the next highest amount of bone formation, followed by the L-SBC and S-SBC sites, in that order, although, again, these amounts were not statistically different.

There was significantly more regenerated bone at 8 weeks after surgery compared to 4 weeks postsurgery in the Bio-Oss group $(P<.05)$. This change was not significant for the other groups $(P>.05)$. No other statistically significant 
differences were observed between the fourth and eighth weeks $(P>.05)$.

\section{DISCUSSION}

In the present study, the amounts of regenerated bone and remaining graft material, along with the severity of inflammation and foreign-body reactions, were compared between groups of defects grafted with S-SBC, L-LBC, or Bio-Oss or left unfilled and allowed to heal for 4 or 8 weeks. The authors observed significantly more inflammation at sites grafted with Bio-Oss compared with the control (ungrafted) group. These findings were consistent with those of other investigators, who observed similar results for Bio-Oss [19, 20]. However, other studies observed no proliferation of inflammatory cells following the application of Bio-Oss for maxillary sinus augmentation and the rehabilitation of other facial skeletal structures [14, 21-25]. These differences could be a result of variations in study designs, sampling techniques, operator accuracy, and histologic criteria. In addition, the present authors found significantly more inflammation in the sites treated with bone ceramic, especially in the L-SBC group, compared to the control and Bio-Oss groups. The release of microparticles from biphasic materials (resulting from insufficient sintering) and the larger particle size of LSBC may have resulted in localized inflammation [26, 27].

With regard to foreign-body responses and the presence of multinucleated giant cells, the grafted sites showed significantly more of a response than the ungrafted sites. L-SBC and Bio-Oss showed the greatest and the least degrees of foreign-body reaction among grafted sites, respectively. The presence of these inflammatory cells could be indicative of a host reaction to inert materials [26]. The semi-osteoclastic multinucleated cells play an important role in the resorption and the substitution of biomaterials.

In the present study, the amount of bone formed in the Bio-Oss-grafted sites was greater than that seen in the other groups, although no statistically significant difference was found. These results are consistent with many previous studies, which have shown Bio-Oss to be a biocompatible material with a good potential for bone formation [10, 11, 28-31]. The porosity and particle shape of this material increase the surface area, which may make Bio-Oss an appropriate scaffold for the penetration of cells mediating osteogenesis and angiogenesis [20]. The control, L-SBC, and S-SBC groups showed less bone formation, in that order. However, again, this difference was not significant. The greater inflammation observed at the bone ceramic-treated sites may have interfered with osteogenesis. The small particle size for the SSBC group may have resulted in compaction of the material, resulting in less space between particles for angiogenesis and therefore less bone formation. The present results showed that there was no significant difference in bone regeneration between the control group and the other groups. This finding is inconsistent with previously published results [16-20]. The authors speculate that the thick periosteal layer of rabbit calvaria, which was carefully sutured during wound closure, may have acted as a natural barrier membrane to induce guided tissue regeneration.

There was a significant difference in the amount of regenerated bone after 8 weeks compared to 4 weeks in the
Bio-Oss group. Also, more secondary bone formation was observed at 8 weeks in the Bio-Oss group. The lower degree of inflammation in the Bio-Oss group may have resulted in less cell damage $[11,32]$, which, in turn, may have contributed to faster bone maturation.

The amount of remaining bone substitute was not significantly different between the study groups. L-SBC showed the highest percentage of remaining graft material, followed by Bio-Oss and S-SBC. The resorption of graft materials by cellular activity is affected by the particle size and the composition and porosity of the material. Larger particles require more time to resorb $[33,34]$. The bone substitutes used in this study had three different sizes: S-SBC, with a particle size of 400 to $700 \mu \mathrm{m}$; L-SBC, with a particle size of 500 to $1000 \mu \mathrm{m}$ and a beta-TCP/HA ratio of 40 to 60 ; and Bio-Oss, with a particle size of 250 to $1000 \mu \mathrm{m}[35,36]$. It is not surprising that the L-SBC had the most remaining material. However, it is surprising that more S-SBC material remained than Bio-Oss, even though the S-SBC particles were somewhat smaller than the Bio-Oss particles. This may be related to the percentage of $\mathrm{HA}$ in the S-SBC, which made its resorption time longer, or it may be related to the different shape of the SBC particles; whereas the SBC particles are round, the Bio-Oss particles are irregularly shaped. This may have resulted in more space between particles, influencing angiogenesis. Also, although Bio-Oss is also available in a 1000 - to $2000-\mu \mathrm{m}$ size, this was considered too large for the rabbit model.

Some studies have shown that larger defects that are filled with HA take longer to be replaced by bone than smaller defects $[33,34,37,38]$. Since the particles had a range of sizes, it is possible that smaller particles in the $S$ SBC filled in around larger particles, eliminating spaces that would otherwise have allowed for vascular and osteogenic ingrowth. The goal of the present study was to compare synthetic bone (Straumann Bone Ceramic, which is composed of HA and beta-TCP and is available in small- and largeparticle versions, both of which are smaller than $1000 \mu \mathrm{m}$ ) with Bio-Oss (natural HA). There is some overlapping of particle sizes in the experimental groups, and this may have contributed to the lack of differences between materials observed here. However, the particle size for both experimental groups was smaller than $1000 \mu \mathrm{m}$. This selection of small particles minimizes the bias that particle size might have on bone formation (i.e., different rates of angiogenesis between the particles). Other studies have showed that differences in particle size influenced the outcome [39]. However, Chackartchi et al. observed, in a histomorphometric analysis, that both granule sizes produced the same pattern of bone formation: bone surrounded the graft granules and produced a network of bridges between graft particles [40]. In addition, the authors considered it more appropriate to use smaller particles because more particles could be utilized to fill the small defects in rabbits, whereas only a few larger particles could have been used.

The HA in Bio-Oss is natural and, unlike the synthetic HA that is used in SBC, may undergo physiologic remodeling and resorption, which promote bone formation. SBC is a bone substitute (a synthetic bone graft material), while BioOss is a xenograft, a deproteinized bovine bone material. A 
study of Park et al. [41] concluded that HA was better than the Bio-Oss as an osteoconductive bone substitute for treating osseous defects in critical-size defects of rat calvaria. Similar findings were observed author when rabbit calvarium was used. In 2008, Schwartz et al. [42] selected bone graft material based on their relative resorption or degradation in vivo and their osteogenic properties. The authors recommended that bone graft materials should be selected not only for their ability to support new bone formation but also for their impact on the remodeling phase of bone healing. With bovine bone mineral, in contrast to synthetic hydroxyapatite and bioactive glass, a larger number of particles were covered with osseous tissue in a histological study of comparison of biomaterial implants in the dental socket [43].

The authors' expectations regarding the higher bone resorption rate of bone ceramic compared to Bio-Oss did not turn out to be correct; perhaps the study time intervals and the number of the samples may have been influential. However, it should be noted that in histomorphometric studies, bony surfaces are detected using a software program and represented as "pixel counts." The development and distribution of the bone components, along with the sum of the bony surfaces, may be one reason that a higher resorption rate for bone ceramic was not found. In the present study, the authors observed a narrow band of bone (Figs. 1C to 1F). Since no space-occupying material existed in this band, the high pixel count may be attributed to software overestimation. The osteogenesis pattern was similar for control defects. In the other groups, however, the thickness of the defects was preserved and new bone was being formed across a broader surface area. Of course, the remainder of the bone substitute material occupied the remaining defect space. This may have resulted in a decreased pixel count of newly formed bone despite the seemingly appropriate thickness of the defect. If more time had been allowed for healing following defect creation and grafting surgery, further resorption of the bone substitute material and its replacement with new bone and greater bone volume may have been achieved in the grafted sites compared to the control group. This absence of more pronounced new bone formation may have been partly a result of dermal and periosteal collapse covering the defects and intracranial pressure, leading to a decreased defect space.

The rabbit is commonly used in animal experiments for medical research. Some of its advantages are that it is easily handled, has a rapid bone turnover rate, and is fully mature within 6 months. Rabbit cranial defects provide a good firstphase bone model for experiments related to bone grafting materials and evaluations of bone regeneration due to the adequate amount of bone marrow facilitating bone formation $[44,45]$. Moreover, the rabbit has a larger cranium than the rat, which makes it possible to create multiple defects in one cranium, which reduces operation time, costs, and observational errors among individuals. The remodeling phase in rabbit is about three times more rapid than in humans; therefore, a healing period of 2 to 4 weeks was considered appropriate to evaluate the early healing response, and 8 weeks or more can be used to assess late healing, such as bone incorporation, resorption of materials, bone remodeling, or the amount of bone regeneration [46-50].
A clinical study of Froum et al. [51] calculated vital bone formation using SBC with biphasic calcium phosphate (BCP) following bilateral sinus grafting. SBC was compared with (Bio-Oss) 6 to 8 months following graft placement. The result was the same as in the present study: The amount of vital bone formation was not significantly different. However, the study was in the human maxillary sinus and the authors waited 6 to 8 months before performing histomorphometric analysis. The bone mixture for each material was composed of $50 \% \quad 0.25$ - to 1.0 -mm-size particles and $50 \%$ 1.0- to 2.0-mm-size particles. Histologically, both materials appeared to be osteoconductive and support new bone formation. Froum et al. did not use a control (i.e., no sinus grafting). However, in the present study there was no statistically significant difference between control, SBC, and BioOss sites. The present authors speculate that if particle sizes with no to minimal overlapping had been chosen, the result might have been different.

Because synthetic HAs and bovine bone are not able to induce bone formation when implanted in subcutaneous muscle tissues and the maxillary defects in rats, these biomaterials are considered to be osteoconductive [52-55]. Osteoconductive materials provide the appropriate scaffold or template to allow vascular ingress, cellular infiltration, and cellular attachment. Osteoinductive materials such as bone morphogenic proteins stimulate uncommitted cells to convert phenotypically to osteoprogenitor cells.

The present study is similar to a recent published clinical study [56]. In which the investigators used qualitative histological and quantitative histomorphical analysis. The extrapolated data from our study showed that both SBC and Bio-Oss are osteoconductive and acceptable bone substitute materials for bone augmentation, which is in agreement with the study of Frenken et al. [56].

\section{CONCLUSIONS}

In the present study in a rabbit grafting model, Straumann Bone Ceramic generated less new bone compared to Bio-Oss and control groups, although the differences in bone formation were not significant. Future studies with a larger sample size and different healing intervals should be designed to further clarify the differences between Bio-Oss and other bone substitutes.

\section{ACKNOWLEDGMENTS}

This study was partly supported by the Dental Research Center, Tehran University of Medical Sciences. The authors would like to thank Dr. M. M. Dehghan for animal testing and for his contributions to the study.

\section{SOURCES OF SUPPORT}

This study was partly supported by the Dental Research Center, Tehran University of Medical Sciences.

\section{ONE-SENTENCE SUMMARY}

Grafting with Straumann Bone Ceramic resulted in less new bone formation compared to Bio-Oss in a rabbit model, although this difference was not significant. 


\section{REFERENCES}

[1] Kauschke E, Rumpel E, Fanghanel J, Bayerlein T, Gedrange T, Proff $\mathrm{P}$. The in vitro viability and growth of fibroblasts cultured in the presence of different bone grafting materials (nanobone and straumann bone Ceramic). Folia Morphol (Warsz) 2006; 65: 37-42.

[2] Bucholz RW. Nonallograft osteoconductive bone graft substitute. Clin Orthop Res 2002; 395: 44-52.

[3] Lynch SE, Genco RJ, Marx RE. Tissue engineering: applications in maxillofacial surgery and periodontics. Carol Stream, IL: Quintessence 1999.

[4] Habibovic P, Kruyt MC, Juhl MV, et al. Comparative in vivo study of six hydroxyapatite-based bone graft substitutes. J Orthop Res 2008; 26: 1363-70.

[5] Fellah BH, Gauthier O, Weiss P, Chappard D, Layrolle P. Osteogenicity of biphasic calcium phosphate ceramics and bone autograft in a goat model. Biomaterials 2008; 29: 1177-88.

[6] Stein JM, Fickl S, Yekta SS, Hoischen U, Ocklenburg C, Smeets R. Clinical evaluation of a biphasic calcium composite grafting material in the treatment of human periodontal intrabony defects: a 12month randomized controlled clinical trial. J Periodontol 2009; 80: 1774-82.

[7] Crawford MJ, Swenson CL, Arnoczky SP, O'Shea J, Ross H. Lyophilization does not inactivate infectious retrovirus in systemically infected bone and tendon allografts. Am J Sports Med 2004 ; 32 (3): 580-6.

[8] Munting E, Wilmart JF, Wijne A, Hennebert P, Delloye C. Effect of sterilization on osteoinduction. Comparison of five methods in demineralized rat bone. Acta Orthop Scand 1988; 59: 34-8.

[9] Anderegg CR, Alexander DC, Freidman M. A bioactive glass particulate in the treatment of molar furcation invasions. J Periodontol 1999; 70: 384-7.

[10] Hammerle CH, Chiantella GC, Karring T, Lang NP. The effect of a deproteinized bovine bone mineral on bone regeneration around titanium dental implants. Clin Oral Implants Res 1998; 9: 151-62.

[11] Nomura T, Katz JL, Powers MP, Saito C. Evaluation of the micromechanical elastic properties of potential bone-grafting materials. J Biomed Mater Res B Appl Biomater 2005; 73: 29-34.

[12] Orsini G, Scarano A, Degidi M, Caputi S, Iezzi G, Piattelli A. Histological and ultrastructural evaluation of bone around Bio-Oss particles in sinus augmentation. Oral Dis 2007; 13: 586-93.

[13] Simion M, Fontana F, Rasperini G, Maiorana C. Vertical ridge augmentation by expanded-polytetrafluoroethylene membrane and a combination of intraoral autogenous bone graft and deproteinized anorganic bovine bone (Bio Oss). Clin Oral Implants Res 2007; 18: 620-9.

[14] Victoria EC, Gnanam FD. Synthesis and characterization of biphasic calcium phosphate. Trends Biomater Artif Organs 2002; 16(1): 12-14.

[15] Paknejad M, Emtiaz S, Rokn A, Islamy B, Safiri A. Histologic and histomorphometric evaluation of two bone substitute materials for bone regeneration: an experimental study in sheep. Implant Dent 2008; 17: 471-9.

[16] Farina NM, Guzon FM, Pena ML, Cantalapiedra AG. In vivo behaviour of two different biphasic ceramic implanted in mandibular bone of dogs. J Mater Sci Mater Med 2008; 19: 1565-73.

[17] Jafarian M, Eslaminejad MB, Khojasteh A, et al. Marrow-derived mesenchymal stem cells-directed bone regeneration in the dog mandible: a comparison between biphasic calcium phosphate and natural bone mineral. Oral Surg Oral Med Oral Pathol Oral Radiol Endod 2008; 105: e14-24.

[18] Bodde EW, Wolke JG, Kowalski RS, Jansen JA. Bone regeneration of porous beta-tricalcium phosphate (Conduit TCP) and of biphasic calcium phosphate ceramic (Biosel) in trabecular defects in sheep. $\mathrm{J}$ Biomed Mater Res A 2007; 82: 711-22.

[19] Schwarz F, Herten M, Ferrari D, et al. Guided bone regeneration at dehiscence-type defects using biphasic hydroxyapatite + beta tricalcium phosphate (Bone Ceramic) or a collagen-coated natural bone mineral (Bio-Oss Collagen): an immunohistochemical study in dogs. Int J Oral Maxillofac Surg 2007; 36: 1198-206.

[20] Artzi Z, Tal H, Dayan D. Porous bovine bone mineral in healing of human extraction sockets. Part 1: histomorphometric evaluations at 9 months. J Periodontol 2000; 71: 1015-23.

[21] Fuerst G, Tangl S, Gruber R, Gahleitner A, Sanroman F, Watzek $\mathrm{G}$. Bone formation following sinus grafting with autogenous bone- derived cells and bovine bone mineral in minipigs: preliminary findings. Clin Oral Implants Res 2004; 15: 733-40.

[22] Iezzi G, Scarano A, Mangano C, Cirotti B, Piattelli A. Histologic results from a human implant retrieved due to fracture 5 years after insertion in a sinus augmented with anorganic bovine bone. $\mathrm{J} \mathrm{Pe}$ riodontol 2008; 79: 192-8.

[23] Merkx MA, Maltha JC, Freihofer HP. Incorporation of composite bone implants in the facial skeleton. Clin Oral Implants Res 2000; 11: 422-9.

[24] Piattelli M, Favero GA, Scarano A, Orsini G, Piattelli A. Bone reactions to anorganic bovine bone (Bio-Oss) used in sinus augmentation procedures: a histologic long-term report of 20 cases in humans. Int J Oral Maxillofac Implants 1999;14: 835-40.

[25] Scarano A, Pecora G, Piattelli M, Piattelli A. Osseointegration in a sinus augmented with bovine porous bone mineral: histological results in an implant retrieved 4 years after insertion: a case report. J Periodontol 2004; 75: 1161-6.

[26] Develioglu H, Saraydin SU, Dupoirieux L, Sahin ZD. Histological findings of long-term healing of the experimental defects by application of a synthetic biphasic ceramic in rats. J Biomed Mater Res A $2007 ; 80: 505-8$.

[27] Lu J, Blary MC, Vavasseur S, Descamps M, Anselme K, Hardouin P. Relationship between bioceramics sintering and micro-particlesinduced cellular damages. J Mater Sci Mater Med 2004; 15: 361-5.

[28] Sculean A, Stavropoulos A, Windisch P, Keglevich T, Karring T, Gera I. Healing of human intrabony defects following regenerative periodontal therapy with a bovine-derived xenograft and guided tissue regeneration. Clin Oral Investig 2004; 8: 70-4.

[29] Slotte C, Lundgren D. Augmentation of calvarial tissue using nonpermeable silicone domes and bovine bone mineral: an experimental study in the rat. Clin Oral Implants Res 1999; 10: 468-76.

[30] Stavropoulos A, Kostopoulos L, Nyengaard JR, Karring T. Deproteinized bovine bone (Bio-Oss) and bioactive glass (Biogran) arrest bone formation when used as an adjunct to guided tissue regeneration (GTR): an experimental study in the rat. J Clin Periodontol 2003; 30: 636-43.

[31] Stephan EB, Jiang D, Lynch S, Bush P, Dziak R. Anorganic bovine bone supports osteoblastic cell attachment and proliferation. J Periodontol 1999; 70: 364-9.

[32] Lu J, Tang T, Ding H, Dai K. [Micro-particles of bioceramics could cause cell and tissue damage]. Sheng Wu Yi Xue Gong Cheng Xue Za Zhi 2006; 23: 85-9.

[33] Crespi R, Cappare P, Gherlone E. Magnesium-enriched hydroxyapatite compared to calcium sulfate in the healing of human extraction sockets: radiographic and histomorphometric evaluation at 3 months. J Periodontol 2009; 80: 210-18.

[34] Francis MD, Ferguson DL, Tofe AJ, Bevan JA, Michaels SE. Comparative evaluation of three diphosphonates: in vitro adsorption (C- 14 labeled) and in vivo osteogenic uptake (Tc-99m complexed). J Nucl Med 1980; 21: 1185-9.

[35] Dietze S, Bayerlein T, Proff P, Hoffmann A, Gedrange T. The ultrastructure and processing properties of straumann bone ceramic and nanobone. Folia Morphol (Warsz) 2006; 65: 63-5.

[36] Richardson CR, Mellonig JT, Brunsvold MA, McDonnell HT, Cochran DL. Clinical evaluation of Bio-Oss: a bovine-derived xenograft for the treatment of periodontal osseous defects in humans. J Clin Periodontol 1999; 26: 421-8.

[37] Sena LA, Caraballo MM, Rossi AM, Soares GA. Synthesis and characterization of biocomposites with different hydroxyapatitecollagen ratios. J Mater Sci Mater Med 2009 ; 20(12): 2394-400.

[38] Oktar FN, Kesenci K, Piskin E. Characterization of processed tooth hydroxyapatite for potential biomedical implant applications. Artif Cells Blood Substit Immobil Biotechnol 1999; 27: 367-79.

[39] Irokawa D, Ota M, Yamamoto S, Shibukawa Y, Yamada S. Effect of $\beta$ tricalcium phosphate particle size on recombinant human platelet-derived growth factor-BB-induced regeneration of periodontal tissue in dog. Dent Mater J 2010 . [Epub ahead of print].

[40] Chackartchi T, Iezzi G, Goldstein M, et al. Sinus floor augmentation using large $(1-2 \mathrm{~mm})$ or small $(0.25-1 \mathrm{~mm})$ bovine bone mineral particles: a prospective, intra-individual controlled clinical, micro-computerized tomography and histomorphometric study. Clin Oral Implants Res 2011; 22: 473-480.

[41] Park JW, Jang JH, Bae SR, An CH, Suh JY. Bone formation with various bone graft substitutes in critical-sized rat calvarial defect. Clin Oral Implants Res 2009; 20(4): 372-8. 
[42] Schwartz Z, Doukarsky-Marx T, Nasatzky E, et al. Differential effects of bone graft substitutes on regeneration of bone marrow. Clin Oral Implants Res 2008; 19(12): 1233-45.

[43] Santos FA, Pochapski MT, Martins MC, Zenóbio EG, Spolidoro LC, Marcantonio E Jr. Comparison of biomaterial implants in the dental socket: histological analysis in dogs. Clin Implant Dent Relat Res 2010; 12(1): 18-25.

[44] Newman E, Turner AS, Wark JD. The potential of sheep for the study of osteopenia: current status and comparison with other animal models. Bone 1995; 16: 277S-84S.

[45] Castaneda S, Largo R, Calvo E, et al. Bone mineral measurements of subchondral and trabecular bone in healthy and osteoporotic rabbits. Skeletal Radiol 2006; 35: 34-41.

[46] Xu S, Lin K, Wang Z, et al. Reconstruction of calvarial defect of rabbits using porous calcium silicate bioactive ceramics. Biomaterials 2008; 29: 2588-96.

[47] Cavalcanti SC, Pereira CL, Mazzonetto R, de Moraes M, Moreira RW. Histological and histomorphometric analyses of calcium phosphate cement in rabbit calvaria. J Craniomaxillofac Surg 2008; 36: 354-9.

[48] Lundgren D, Nyman S, Mathisen T, Isaksson S, Klinge B. Guided bone regeneration of cranial defects, using biodegradable barriers: an experimental pilot study in the rabbit. J Craniomaxillofac Surg 1992; 20: 257-60.

[49] Torres J, Tamimi FM, Tresguerres IF, et al. Effect of solely applied platelet- rich plasma on osseous regeneration compared to Bio-
Oss: a morphometric and densitometric study on rabbit calvaria. Clin Implant Dent Relat Res 2008; 10: 106-12.

[50] Sohn JY, Park JC, Um YJ, et al. Spontaneous healing capacity of rabbit cranial defects of various sizes. J Periodontal Implant Sci 2010; 40: 180-7.

[51] Froum SF, Wallace SS, Ech SC, Elian N, Tarnow, DP. Histomorphometric comparison of a biphasic bone ceramic to anorganic bovine bone for sinus augmentation: 6- to 8-month postsurgical assessment of vital bone formation. A pilot study. Int J Periodontics Restorative Dent 2008; 28: 273-81.

[52] Cruz AC, Pochapski MT, Daher JB, Silva JC, Pilatti GL, Santos FA. Physicochemical characterization and biocompatibility evaluation of hydroxyapatites. J Oral Sci 2006; 48: 219-26.

[53] Pinholt EM, Bang G, Hannaes HR. Alveolar ridge augmentation in rats by Bio-Oss. Scand J Dent Res 1991; 99: 154-61.

[54] Cardaropoli G, Araújo M, Hayacibara R, Sukekava F, Lindhe J. Healing of extraction sockets and surgically produced:augmented and non-augmented defects in the alveolar ridge: an experimental study in the dog. J Clin Periodontol 2005; 32: 435-40.

[55] Indovina A Jr, Block MS. Comparison of 3 bone substitutes in canine extraction sites. J Oral Maxillofac Surg 2002; 60: 53-8.

[56] Frenken JWFH, Bouwman WF, Bravenboer N, Zijderveld SA, Schulten EAJM, Ten Bruggenkate CM. The use of Straumann Bone Ceramic in a maxillary sinus floor elevation procedure: a clinical, radiological, histological and histomorphometric evaluation with a 6-month healing period. Clin Oral Implants Res 2010; 21(2): 201-8

(C) Rokn et al.; Licensee Bentham Open .

This is an open access article licensed under the terms of the Creative Commons Attribution Non-Commercial License (http://creativecommons.org/licenses/by-nc/3.0/) which permits unrestricted, non-commercial use, distribution and reproduction in any medium, provided the work is properly cited. 